\title{
Why is it Hard to Induce Long-Term Depression?
}

\author{
Lubica Benuskova, Member, IEEE \\ Department of Computer Science \\ University of Otago \\ Dunedin, New Zealand \\ Email: lubica@cs.otago.ac.nz
}

\begin{abstract}
Most of the interest in computational modelling is devoted to the phenomenon of long-term potentiation (LTP) of synaptic efficacy. Long-term depression (LTD) of synaptic connections is often overlooked. We have found that it is in fact very difficult to induce LTD experimentally and the conditions for LTD induction are much less clear than those for induction of LTP. We concentrate on granule cells in the hippocampal dentate gyrus and using the spike-timing-dependent plasticity (STDP) rule with metaplasticity we show that to evoke homosynaptic LTD or depotentiation of previously potentiated synapses in the spiking model of granule cell is not easy similarly like in a number of experimental studies. On the other hand, heterosynaptic LTD, which accompanies homosynaptic LTP is induced readily both in the model as well as in experiments. We offer possible explanation of these phenomena from STDP, metaplasticity and spontaneous activity.
\end{abstract}

Keywords-computational neuroscience; synaptic plasticity; spike-timing dependent plasticity; metaplasticity

\section{INTRODUCTION}

The ability of high-frequency stimulation (HFS) to induce LTP at all major excitatory synaptic pathways in hippocampus is well-characterized [1], [2], [3]. There are however, mixed reports on ability of low-frequency stimulation (LFS) to induce LTD on naive synapses and depotentiation of previously potentiated ones. In this paper, we will concentrate on granule cells in the hippocampal dentate gyrus. The entorhinal cortex projects monosynaptically to the granule cells in the dentate gyrus via the lateral and medial perforant paths. Medial perforant path (MPP) and lateral perforant path (LPP) are two separate inputs terminating on separate but adjacent distal dendritic zones of the hippocampal dentate granule cells. They together form an ipsilateral perforant path input (PP) [4], [5]. We choose granule cells in the hippocampal dentate gyrus for two main reasons. Firstly, there are number of studies reporting failure to induce homosynaptic LTD and depotentiation in the perforant path in awake or anesthetized rats of all ages [6]-[8]. Still, some studies found that depotentiation can be achieved under certain timing conditions between HFS and LFS [8]. Secondly, it has been shown that heterosynaptic LTD can be reliably induced in the lateral perforant pathway, when the medial perforant pathway receives HFS [1] or vice versa, the heterosynaptic LTD is induced in the medial perforant path when the lateral perforant path receives HFS [2]. Thus, there are some puzzling data on LTD in the dentate gyrus, namely why it would be difficult to elicit homosynaptic LTD, if the same input readily expresses heterosynaptic LTD when the neighboring input pathway is tetanized?

There are numerous experimental studies showing that both LTP and LTD depend not only on the frequency of the presynaptic stimulation but also on the precise timing of preand postsynaptic spikes. This property is called the spiketiming dependent plasticity or STDP [9], [10]. In 2006, Lin et al. [11] published results of the study of the STDP in granule cells in hippocampal slices. They used the pairs of presynaptic stimulation of LPP and the postsynaptic antidromic spikes delivered to granule cells in different orders and with different delays to succesfully induce STDP. Synapses of the LPP exhibited STDP with two windows, one for LTP for the prepost sequence and the other one for LTD for the post-pre sequence. In addition, Lin et al. [11] showed the interaction of STDP and frequency-dependent plasticity at one synaptic path thus suggesting they may actually share the same mechanisms.

Benuskova and Abraham used the computational model of STDP to explain the frequency-dependent homosynaptic LTP and heterosynaptic LTD in the dentate gyrus [12]. In their model, synapses of MPP and LPP modify according to the pair-wise STDP rule, in which the amplitudes of LTP and LTD are not constant but instead depend metaplastically on the postsynaptic spike count averaged over some recent past. This way Benuskova and Abraham [12] linked the STDP rule to the sliding property of LTD/LTP threshold in the BCM theory [13]. In this new formulation of the STDP rule, the relative size of LTP is larger and LTD smaller when the average postsynaptic activity is low, thus favouring LTP, whereas the magnitude of LTP is smaller and LTD larger when the average postsynaptic activity is high, thus favouring LTD. Additional assumption in their study was that of an ongoing spontaneous spiking activity along the MPP and LPP inputs in vivo. This was a crucial feature of Benuskova and Abraham's model. They concluded from their model that heterosynaptic LTD is in fact a homosynaptic phenomenon that requires an ongoing spontanepous presynaptic spiking activity to occur. This prediction was experimentally tested and confirmed by Abraham et al. [14]. Abraham et al. [14] blocked the ongoing spontaneous spiking activity by injecting the anesthetic procaine to the LPP and then they tetanised MPP. This protocol induced homosynaptic LTP in tetanized MPP but no heterosynaptic LTD in LPP with no spontaneous activity.

Thus, in this paper we simulate the same model as in 
Benuskova and Abraham [12], which successfully explained heterosynaptic LTD, but this time with stimulation protocols that failed to induce homosynaptic LTD [6]-[8] to see whether the model can reproduce these data and if yes, then what is the explanation it can offer for the difficulties in evoking homosynaptic LTD.

\section{Methods}

\section{A. Neuron Model}

We use a simple spiking model of a hippocampal dentate granule cell, in which we consider only excitatory inputs. Thus, a model cell has $N=3$ excitatory inputs, two of them representing ipsilateral medial and ipsilateral lateral perforant paths, MPP and LPP, respectively, and the third excitatory input representing the commissural/associational input. The commissural/associational fibres originate from the mossy cells in the contralateral and ipsilateral hilar region and terminate in the proximal dendritic zone of the granule cell dendritic tree [5].

To model a granule cell we employ the simple model of spiking neuron introduced by Izhikevich [15], which can simulate the wide range of spiking behaviours by varying the values of just four parameters $a, b, c, d$ [15], [16]. In this simple spiking model, the dimensionless variable $v$ represent the membrane potential of the neuron and $u$ represent a membrane recovery variable, which provides a negative feedback to $v$. The dynamics of $v$ and $u$ is described by the following set of ordinary differential equations:

$$
\begin{gathered}
\dot{v}=0.04 v^{2}+5 v+140-u+\text { Input } \\
\dot{u}=a(b v-u)
\end{gathered}
$$

Synaptic inputs are delivered via the variable Input. Our model granule cell has $N=3$ excitatory inputs, two of them representing ipsilateral medial and ipsilateral lateral perforant paths, MPP and LPP, respectively, and the third excitatory input representing the commissural/associational input (denoted as ComAs). Total synaptic input corresponding to variable Input thus reads:

$$
\text { Input }=\sum_{k=1}^{N} s_{k} w_{k} I_{k}
$$

where $k=$ MPP, LPP, ComAs; $s_{k}=1$ or 0 when a presynaptic spike is present or absent at a given input, respectively; $w_{k}$ is the weight of the given input synapse, and $I_{k}$ is the intensity of electric stimulus delivered to the given pathway. Intensity of electric stimulus is understood as the number of input fibers/synapses that are engaged within a stimulated pathway, and thus is dimensionless. The stimulus intensity variable was introduced because in some simulated experiments, experimenters might use different intensities for conditioning and for testing stimulation.

The firing threshold of granule cells is equal to $24 \mathrm{mV}$ [4]. When $v$ crosses the firing threshold from below, the granule cell fires a postsynaptic spike. After the postsynaptic spike is fired, after $1 \mathrm{~ms}$, in the next iteration, the membrane voltage $v$ and the recovery variable $u$ are reset as follows:

$$
v \leftarrow c, \quad u \leftarrow u+d
$$

The model neuron cannot fire again for a few miliseconds thus effectively simulating the refractory period. Since granule cells are regularly spiking cells, we simulate the simple spiking model with the values of parameters $a, b, c, d$ corresponding to a regularly spiking cell, i.e. $a=0.02, b=0.2, c=-69 \mathrm{mV}, d$ $=2$ [15], [16]. The model is simulated in real time with the time step of $1 \mathrm{~ms}$, but with the integration step of $0.5 \mathrm{~ms}$ for numerical stability as in the code in [15].

\section{B. Simulated Spontaneous Spiking}

Because the simulated experiments were done in live animals, our model granule cell was subject to simulated ongoing spontaneous input activity that is present in biological neurons in vivo. All the spontaneous input spiking was generated randomly as Poisson spike trains. Ongoing spontaneous input spiking had two components. The first component were the correlated spontaneous spikes in MPP and LPP and commissural/associational input. By correlated we mean that the spikes occurred at the same time in all three inputs. Superimposed on this correlated spiking was uncorrelated random activity occuring at different times in all three inputs. The average frequency of all spontaneous input spiking at each of the three inputs was $8 \mathrm{~Hz}$ to simulate the theta rhythm modulation observed experimentally during the awake state of the rat [17], [18]. Out of this $8 \mathrm{~Hz}$, we tried different proportions of correlated versus uncorrelated components, e.g. correlated spikes with average frequency of $7 \mathrm{~Hz}$ accompanied with uncorrelated spikes at frequency of $1 \mathrm{~Hz}$ or correlated spikes with average frequency of $1 \mathrm{~Hz}$ accompanied with uncorrelated spikes at frequency of $7 \mathrm{~Hz}$, and all the options in between these two extremes. The simulated spontaneous activities as described above have lead to a postsynaptic spontaneous spiking activity of the simulated granule cell of $\sim 1$ $\mathrm{Hz}$, which is in accordance with the data [19]. All described scenarios of presynaptic spontaneous spiking activity lead to the same results as described in the Results section below.

In addition, all types of input-output spiking activity, i.e. spontaneous or evoked by various protocols (see below), were accompanied by the STDP with metaplasticity as described in the next subsection.

\section{Synaptic Plasticity Rule}

In our model, we assume that classical pair-based STDP underlies all frequency-evoked synaptic plasticity, although dependence of STDP on more spikes was recently suggested [20]. However, Lin et al. [11] used the two spike-pairing paradigm in the dentate gyrus to evoke STDP and identified two exponetial windows, one for LTP and the other one for LTD. Because we have no evidence about interactions of more than two spikes in granule cells, each excitatory synaptic weight modifies according to the additive nearest neighbors 
STDP rule using the standard exponential relationships as in [21]:

$$
\begin{array}{llll}
\Delta w_{+}=A_{+} \exp \left(-\Delta t / \tau_{+}\right) & \text {for } & \Delta t>0 \\
\Delta w_{-}=A_{-} \exp \left(-\Delta t / \tau_{-}\right) & \text {for } & \Delta t<0
\end{array}
$$

where $\Delta t=t_{\text {post }}-t_{\text {pre }}$ is the time difference between the post- and presynaptic spikes; $\tau_{+}$and $\tau_{-}$are decay constants of windows for synaptic potentiation and depression, respectively. Synaptic change is comprised of contributions from only two nearest postsynaptic spikes. So for each presynaptic spike, only two postsynaptic spikes are considered: the one that occurs right before and the one that occurs right after the given presynaptic spike, i.e.

$$
\Delta w(t+\delta t)=w(t)\left(1+\Delta w_{+}-\Delta w_{-}\right)
$$

where $\delta t=1 \mathrm{~ms}$ is the time step of weight updating. the reason for the nearest neighbors implementation of STDP is that Izhikevich and Desai [21] showed that this implementaion leads to the BCM-like LTD/LTP threshold value for the frequency of presynaptic stimulation.

Benuskova and Abraham [12] showed that in order to account for synaptic plasticity in the dentate gyrus when the spontaneous activity is going on, it is necessary to introduce metaplasticity into the STDP rule. They proposed that the amplitudes of positive and negative synaptic changes, $A_{+}$and $A_{-}$, are not constant anymore, but instead they depend on the average of the postsynaptic spiking activity over some recent past $\langle c\rangle_{\tau}$, calculated as an integral:

$$
\langle c(t)\rangle_{\tau}=\frac{c_{0}}{\tau} \int_{-\infty}^{t} c\left(t^{\prime}\right) \exp \left(-\left(t-t^{\prime}\right) / \tau\right) d t^{\prime}
$$

with $c(t)=1$ or 0 if the postsynaptic spike is present or absent at time $t$, respectively, $\tau$ is the integration period, and $c_{0}$ is the scaling constant. The integral can be replaced with the discrete sum, but we actually numerically calculated the above integral in our code. The rationale for using the spike count for equation (7) comes from experiments of Abraham et al. [1], in which antidromic spikes with NMDA receptors blocked were sufficient to increase the threshold for subsequent LTP induction by HFS. In this calculation of the average of the past spike count of the postsynaptic cell, the most recent spikes have the largest weight and this influence decays exponentially into the past. This relationship was inspired by the calculation of the current position of the LTD/LTP threshold in the plasticity model of the visual [22] and somatosensory cortices [23]. Thus, the amplitudes of potentiation and depression windows for the STDP equations metaplastically expand or shrink as a function of previous postsynaptic activity like this:

$$
A_{+}(t)=\frac{A_{+}(0)}{\langle c\rangle_{\tau}} \quad \text { and } \quad A_{-}(t)=A_{-}(0)\langle c\rangle_{\tau}
$$

Positive constants $A_{+}(0)$ and $A_{-}(0)$ are initial amplitude values for synaptic potentiation and depression, respectively, used in the simulations. If $\langle c(t)\rangle_{\tau}=0$ then $A_{+}(t)=A_{+}(0)$ and $A_{-}(t)=A_{-}(0)$. Equations (8) simply mean the amplitude for LTP gets smaller and the LTD amplitude gets larger when the average postsynaptic activity is high. The opposite is true for a low average postsynaptic activity. Then, it is easier to potentiate the synapses than to weaken them due to an expanded amplitude for LTP and shrunken amplitude for LTD. The new values of $A_{+}(t)$ and $A_{-}(t)$ are updated at each iteration based on the actual value of the recent average activity $\langle c(t)\rangle_{\tau}$.

Values of model parameters used in the following computer simulations were: $A_{+}(0)=0.001, A_{-}(0)=0.01, \tau_{+}=20 \mathrm{~ms}$, $\tau_{-}=100 \mathrm{~ms}, \tau=60 \mathrm{sec}, c_{0}=1000$. Unless stated otherwise, intensities of evoked and spontaneous presynaptic spikes were equal for all pathways, i.e. $I_{\mathrm{MPP}}=I_{\mathrm{LPP}}=I_{\mathrm{ComAs}}=$ 150. Initial values of synaptic weights were chosen to be $w_{\mathrm{MPP}}(0)=w_{\mathrm{LPP}}(0)=w_{\mathrm{ComAs}}(0)=0.033$, so when the three input pathways were spiking simultaneously in a close temporal succession, a postsynaptic spike followed after 2$3 \mathrm{~ms}$, which corresponds to the delay in real granule cells between their synaptic stimulation and natural postsynaptic spiking. The same results were obtained with stimulus intensity $=100$ and initial weights $=0.05$, or stimulus intensity $=$ 200 and initial weights $=0.025$.

It is notable that in our simulations, we used no lower nor upper bound on synaptic weights, no renormalisation, no decay term, etc. It is only the dynamics of changing LTD and LTP amplitudes that prevents the weight values to grow to infinity.

\section{RESULTS}

First we simulated the granule cell just in the condition of an ongoing spontaneous presynaptic activity as described in subsection 2.2. The first $30 \mathrm{~min}$ in each of the following figures shows a dynamically stable trace achieved for the model granule cell for the spontaneous input activity and the choice of the cell and synaptic plasticity parameters given in section 2. Parameter values were optimized by hand to give the model cell stability in the spontaneous activity condition, and to achieve the best match with the experimental data on homosynaptic LTD and depotentiation studies. The figures below are for input spontaneous correlated spikes with average frequency of $7 \mathrm{~Hz}$ accompanied with uncorrelated spikes at frequency of $1 \mathrm{~Hz}$.

To study the induction of LTD in our computer simulations of the model granule cell we used exactly the same protocols as in Abraham et al. [7] who studied homosynaptic LTD and depotentiation in the rat dentate gyrus in vivo. First they applied LFS to naive, i.e. unstimulated, perforant path, which is a mixture of MPP and LPP fibers. LFS in the real experiment and in our simulations consisted of 100 pulses of $1 \mathrm{~Hz}$, and then of 900 pulses of $1 \mathrm{~Hz}$ or 900 pulses of $3 \mathrm{~Hz}$. They also gave the test pulses $(1 / 20 \mathrm{sec})$ for $10 \mathrm{~min}$ before and 20-30 min after LFS to monitor the amplitude of excitatory postsynapytic potentials before and after LFS. Intensity of test and LFS stimuli was the same. In our model, the LFS and test stimuli were administered with exactly the same timing as 

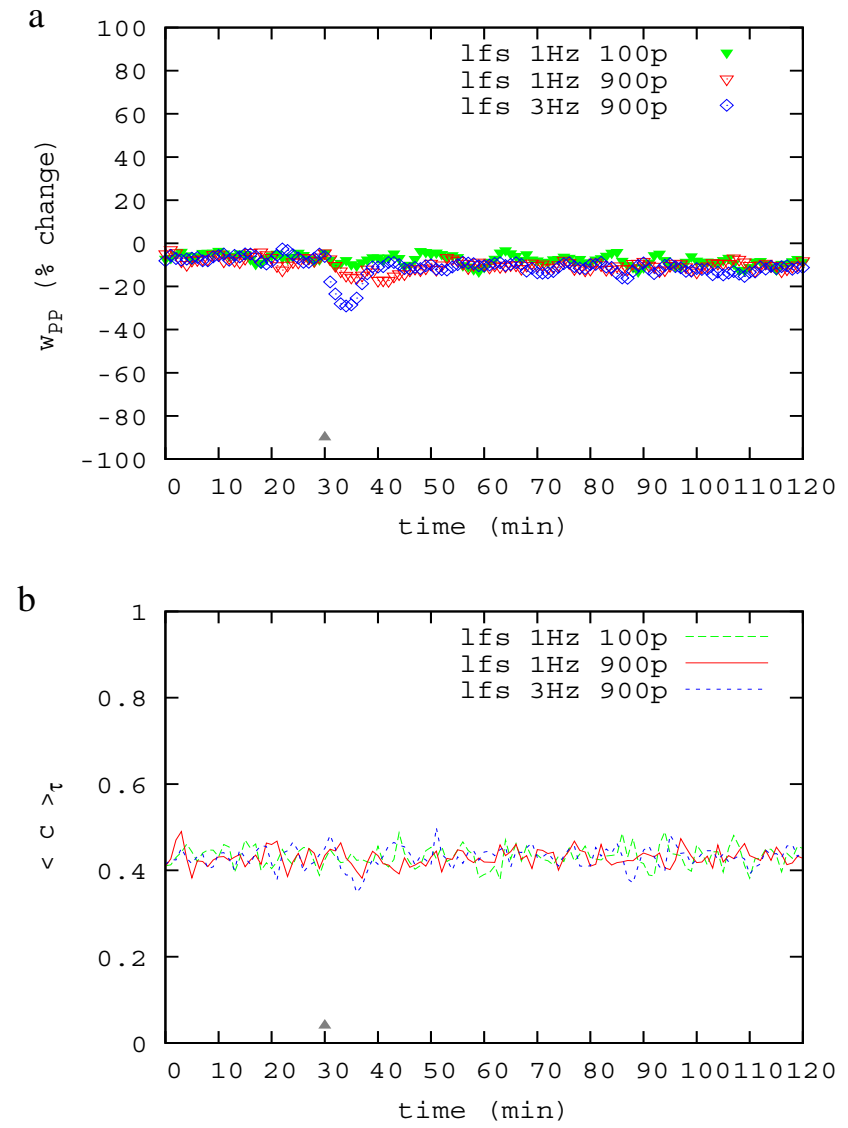

Fig. 1. (a) Evolution of weights of the PP input to model granule cell before, during and after LFS delivered to PP. LFS to PP commenced at 30 min (arrowhead) and lasted till $45 \mathrm{~min}$ of time. (b) Corresponding evolution of the average postsynaptic spike count over time. All traces in the graphs are averages of 10 runs.

in the experiment while the spontaneous presynaptic activity as described in the previous section was present all the time. We also simulated the administration of test stimuli although we do not need them as we can monitor the weight changes directly. We also simulated the model without the test stimuli, and the results were the same. Thus the test stimuli in the model do not make any difference, which should be the case.

The results for these three LFS protocols are shown in Figure 1. As PP is the mixture of MPP and LPP, we consider $w_{\mathrm{PP}}=w_{\mathrm{MPP}}+w_{\mathrm{LPP}}$ and the pictures depict the percentual change against the initial steady state weight value. We can see that the LFS induces only transient depression of PP input weights. Temporary depression of the PP weights lasts only for the period of LFS, then the weights return to the pre-LFS control values. The same magnitude and course of transient depression was observed in real experiment (Figures $1 \mathrm{~B}$ and $3 \mathrm{~A}$ in [7]). We do not show the S.D. for clarity of the pictures, just the averages of 10 runs. S.D. for each weight at each time instant was never larger than $8 \%$ of the initial steady state weight value, i.e. it was 0.0024 at maximum, while the initial weight value of single input was 0.033 . That was the case for all subsequent experiments as well. In case of LFS

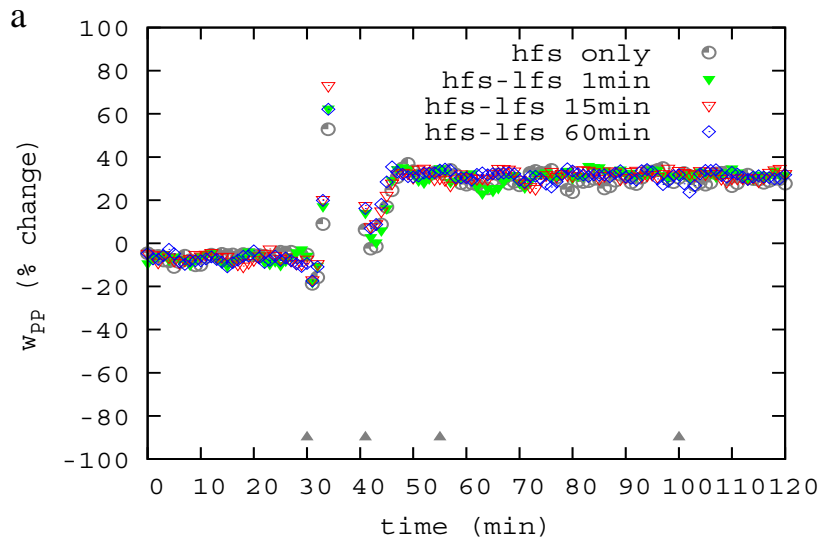

b

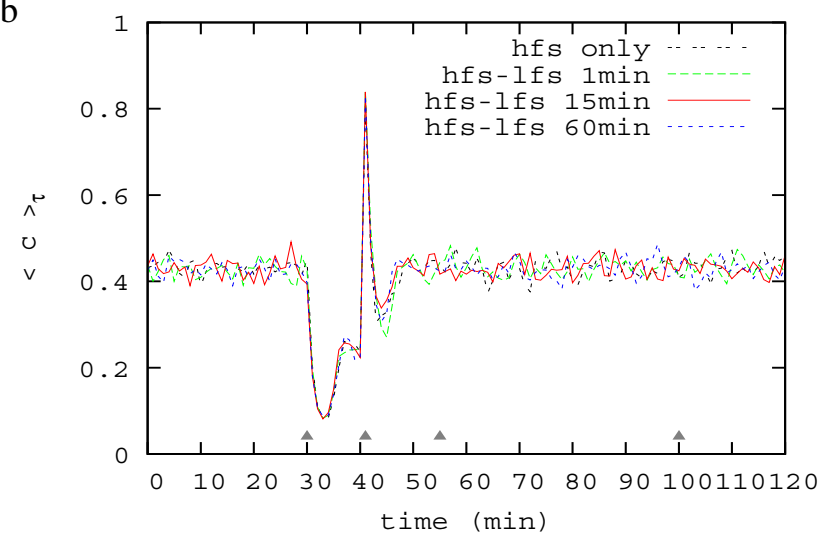

Fig. 2. (a) Evolution of weights of the PP input to model granule cell before, during and after HFS followed by LFS. HFS of PP commenced at 30 min (first arrowhead) and lasted till $40 \mathrm{~min}$ of time. LFS of PP consisiting of 100 pulses of $1 \mathrm{~Hz}$ commenced $1 \mathrm{~min}, 15 \mathrm{~min}$ or $60 \mathrm{~min}$ after the end of HFS (subsequent arrowheads). (b) Corresponding evolution of the average postsynaptic spike count over time. All traces in the graphs are averages of 10 runs.

delivered to PP, there was no change of the weight of the simulated commissural/associational input. These traces are not shown for the sake of Figure 1a clarity. In Figure 1b, we also monitored the evolution of $\langle c(t)\rangle_{\tau}$ as it affects the current size of potentiation and depression (see equation 8). We can see that when the synaptic weight goes down, there is a relatively larger "ripple" in $\langle c(t)\rangle_{\tau}$ after the arrowhead, too. This is caused by the fact that $\langle c(t)\rangle_{\tau}$ is calculated as the average of past postsynaptic spike count. When the input synapses are weakened, there is fewer postsynaptic spikes and thus $\langle c(t)\rangle_{\tau}$ decreases. This decrease is temporary, as it also means a decrease of further depression and increase in the potentiation size, so the balance is shifted towards potentiation and the weights return to control pre-LFS values, and so does the average postsynaptic spiking $\langle c(t)\rangle_{\tau}$.

In the next set of computer experiments (Figure 2), like in the real ones [7], HFS was applied to PP to induce homosynaptic LTP of PP to study depotentiation. HFS consisted of 50 trains of ten pulses at $400 \mathrm{~Hz}$. HFS was delivered in bursts of 5 trains at 1-sec intervals, with $60 \mathrm{sec}$ between bursts. Subsequent LFS consisiting of 100 pulses of $1 \mathrm{~Hz}$ was 
delivered to PP at variable intervals after HFS completion, i.e. after $1 \mathrm{~min}, 15 \mathrm{~min}$ and $60 \mathrm{~min}$. The results of this series of simulations are shown in Figure 2. We can see in Figure 2a that HFS induces lasting LTP of the magnitude about $40 \%$ like in the real experiment (see Figure 2A in [7]). Subsequent LFS consisiting of 100 pulses of $1 \mathrm{~Hz}$ delivered to $\mathrm{PP}$ at variable intervals after HFS completion, i.e. after $1 \mathrm{~min}, 15 \mathrm{~min}$ and 60 min does not cause depotentiation, which is the case also in the real experiment (Figure 2A in [7]).

It is interesting to observe the dynamics of the average postsynaptic spike count in Figure 2b. The average spike count actually decreases immediately after the start of HFS. This is due to the refractory period of the model neuron and one additional assumption of the model. This additional assumption states that during the HFS, all the spontaneous spiking at the three inputs gets decorrelated, i.e. spontaneous spikes come at different times at all three inputs. The average frequency of the spontaneous spiking remains the same, i.e. $8 \mathrm{~Hz}$, but the spikes never occur at the same time like they do before and after the HFS. Benuskova and Abraham used the same assumption in their modelling of heterosynaptic plasticity in the dentate gyrus [12]. We assume this decorrelation may happen as a result of artificially delivered HFS. This drop in $\langle c(t)\rangle_{\tau}$ enables the tetanized path to potentiate. The dynamics of $\langle c(t)\rangle_{\tau}$ also exhibits the stabilizing oscillations opposite to the direction of the synaptic change.

In another set of experiments (Figure 3), LFS was delivered 1 min after the end of HFS and consisted either of 100 pulses of $1 \mathrm{~Hz}$ or of 900 pulses of $1 \mathrm{~Hz}$ or 900 pulses of $3 \mathrm{~Hz}$. The results are shown in Figure 3a. None of these protocols caused depotentiation, which is consistent with the experimental data (Figure 4A and 4B in [7]). That is the case when the LFS did not cause the epileptic seizure-like activity of the granule cells, in which case Abraham notes there is an overall granule cell response depression, which manifests like an LTP reversal, but the next day the normal amplitude of LTP was observed (Figure 4 in [7]).

Martin [8] showed that 10 min (3000 pulses) of $5 \mathrm{~Hz}$ cause depotentiation when applied less than 1 min after HFS, but he also notes that $5 \mathrm{~Hz}$ stimulation was always accompanied by seizure-like epileptiform activity of the granule cells. However, what he considered to be a depotentiation might as well been the granule cell response depression as documented by Abraham et al. in a similar stimulation paradigm (900 pulses of $3 \mathrm{~Hz}$ ) [7]. Thus we tried the 3000 pulses of $5 \mathrm{~Hz}$ commenced $10 \mathrm{~s}$ after the end of HFS too; but, without simulation of the epileptiform postsynaptic activity because we would have to enhance our neuron model with some mechanism that causes its overall response depression when it goes into an epileptic mode of activity. Izhikevich and other simple spiking neuron models do not have such a mechanism, so this is something that needs to be taken into account in the future. We can see in Figure $3 b$ that the transient depotentiation lasted only while there was LFS then the weights went back to the potentiated values. Thus our results are in accordance with Abraham et al. [7] and also with Errington et al. [6]. Both these studies used

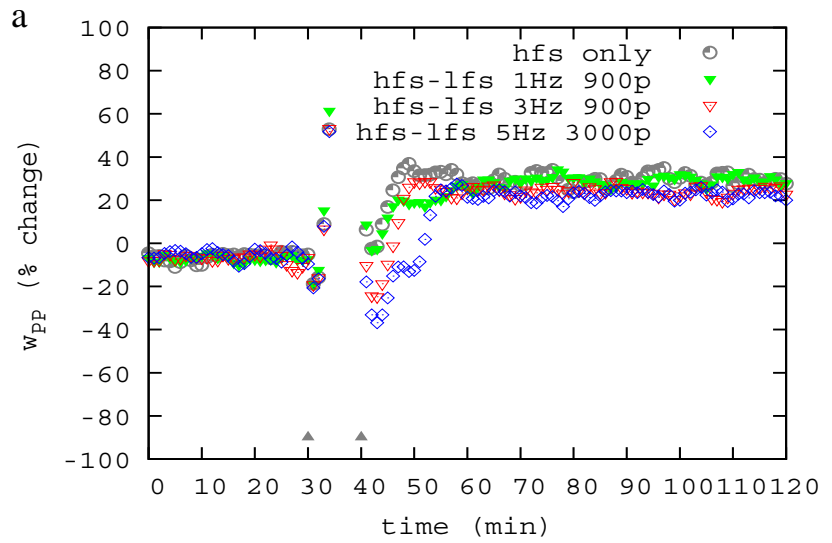

$\mathrm{b}$

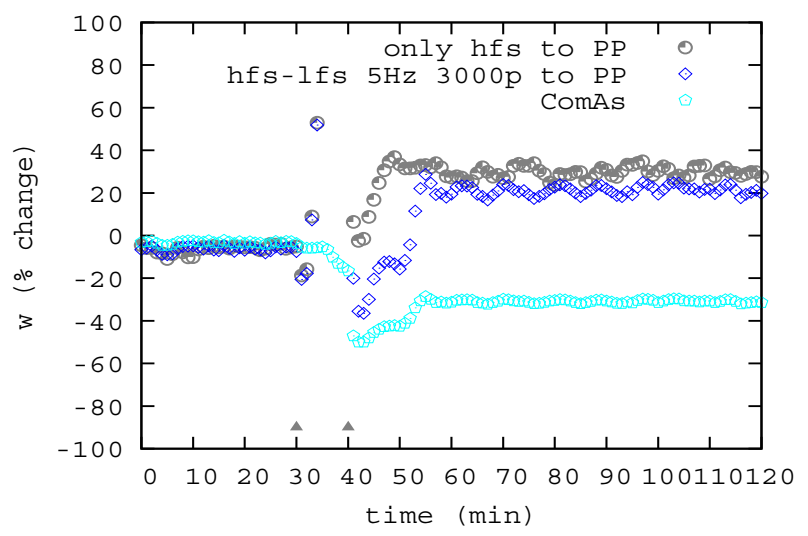

Fig. 3. Evolution of weights of the PP input to model granule cell before, during and after HFS followed by LFS. (a) HFS to PP commenced at $30 \mathrm{~min}$ (first arrowhead) and lasted till 40 min of time. Subsequent LFS commenced 1 min after HFS (second arrowhead) and consisted either of 100 pulses at $1 \mathrm{~Hz}$ or 900 pulses of $1 \mathrm{~Hz}$ or 900 pulses at $3 \mathrm{~Hz}$. (b) HFS to PP commenced at 30 $\mathrm{min}$ (first arrowhead) and lasted till $40 \mathrm{~min}$ of time. LFS commenced $10 \mathrm{~s}$ after HFS (second arrowhead) and consisted of 3000 pulses of $5 \mathrm{~Hz}$. The lowest trace shows the evolution of the weights of the third commissural/associational input, which relayed only spontaneous spiking activity. All traces are average of 10 runs.

similar protocols and showed that various numbers of LFS pulses of the frequency $1-5 \mathrm{~Hz}$ do not cause depotentiation in the dentate gyrus.

It is notable that all instances of homosynaptic LTP of the PP synapses are accompanied by the heterosynaptic LTD of the commissural/associational input as we can see in Figure $3 \mathrm{~b}$, the lowest trace (not included in Figure 2a and Figure 3a for the sake of clarity). This is exactly the same situation to the homosynaptic LTP of MPP being accompanied by the heterosynaptic LTD of LPP in the model of plasticity in the dentate gyrus [12]. As this commissural/associational input was not checked in the cited experiments of Abraham et al. [7], Errington et al. [6], and Martin [8], it is a testable prediction of the current model for these protocols in vivo.

\section{CONClusions}

There is a plethora of different formulations and implementations of the STDP rule (for an extensive review see [24]). The reason for choosing the STDP rule with metaplasticity as 
described in subsection 2.3 is its previous success in modelling homosynaptic LTP and heterosynaptic LTD in the dentate gyrus in vivo [12], [14]. Based on this pair-based STDP, we attemp to gain a novel insight into the homosynaptic LTD phenomenon using a spiking model of granule cell.

In our simulations we used different values of $\tau_{+}$and $\tau_{-}$ than obtained experimentaly in [11]. Lin et al. [11] estimated $\tau_{+}=26 \mathrm{~ms}$ and $\tau_{-}=36 \mathrm{~ms}$. We can obtain qualitatively the same results with their parameter values, however we have used much bigger $\tau_{-}=100 \mathrm{~ms}$ to achieve the best quantitative match with all the simulated experimental data. The reason for this difference might be that Lin et al. [11] used in vitro slices, whereas we modelled the in vivo experiments. The values of STDP parameters may not be the same for in vitro and in vivo conditions.

Heterosynaptic LTD is induced very readily in our model when the other inputs are potentiated by HFS. This is not the case of the homosynaptic LTD, however, which ought to be evoked by LFS. The explanation lies in the STDP rule itself, which we assume is the basis of frequency-dependent synaptic plasticity, too. Let us use the following illustration of the concept, see Figure 4. All the following reasoning holds for the nearest-neighbors implementation of the pair-wise STDP, which we used in our simulations.

Let us first exclude the spontanous activity and consider only LFS. The upper trace in Figure 4a illustrates the succession of presynaptic spikes in LFS. The lower trace illustrates the series of postsynaptic spikes that are evoked by presynaptic spikes. Since the frequency of LFS is between $1 \mathrm{~Hz}$ and $5 \mathrm{~Hz}$, spacing between presynaptic spikes is from $1000 \mathrm{~ms}$ to 200 $\mathrm{ms}$, respectively. It is a huge interval for the LTD window, and thus unless the amplitude of LTD is much much larger than the amplitude of LTP, it would be impossible to induce LTD with such LFS. Even the LTP may follow.

What happens if we included spontaneous pre- and postsynaptic spiking? This situation is illustrated in Figure $4 \mathrm{~b}$ and $4 \mathrm{c}$. The average frequency of input spikes to granule cell is $8 \mathrm{~Hz}$ while the average frequency of spontaneous spiking of granule cell itself is only around $1 \mathrm{~Hz}$ (Figure $4 \mathrm{~b}$ ). Thus, in case of the granule cell there will be 8 spontaneous spikes between each two evoked presynaptic spikes (if the frequency of LFS was $1 \mathrm{~Hz}$ ). However, as for the postsynaptic spikes, there will be at most one spontaneous postsynaptic spike within this window. Thus, it seems that for the nearest neighbors pairwise STDP the mutual LTD/LTP interactions may as well annul itself with zero overall synaptic change.

It seems that it would be impossible to induce homosynaptic LTD at all, which is not the case, for example in the hippocampal area CA1 in vivo [3]. CA1 neurons have a higher spontaneous output activity than granule cells, on the order of $8 \mathrm{~Hz}$ [17]. This spontaneous postsynaptic spiking would place more spikes between the two postsynaptic spikes evoked by LFS (see Figure 4c). If we consider the nearestneighbors implementation of STDP, more postsynaptic spikes would actually help LTD because they are more close to the presynaptic spikes and thus the overall LTD size is larger than a
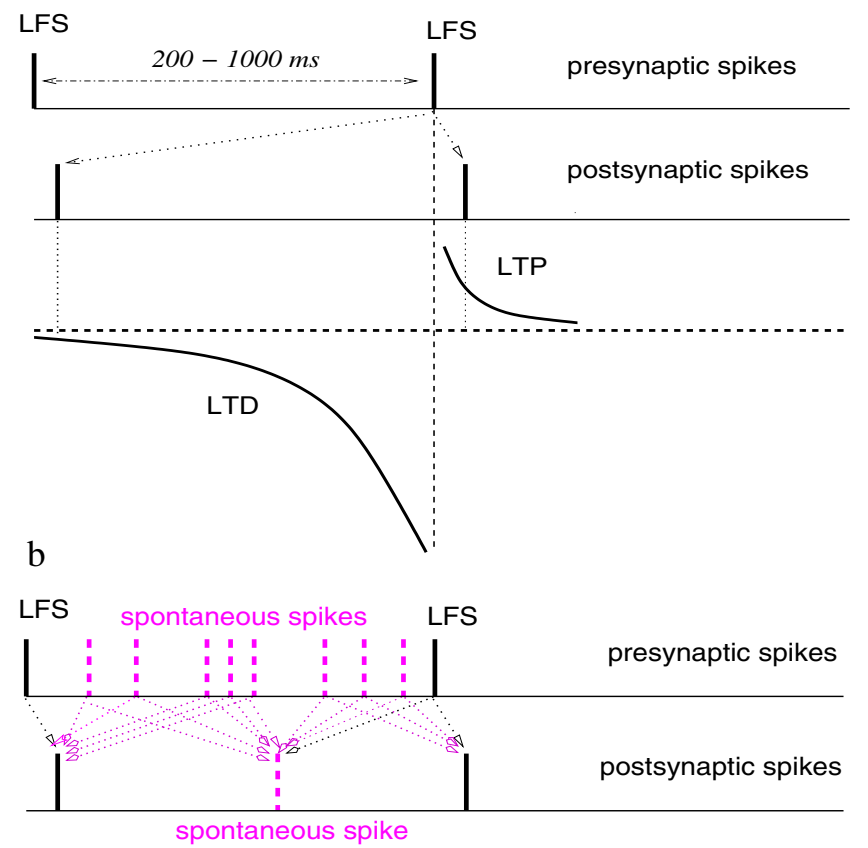

c

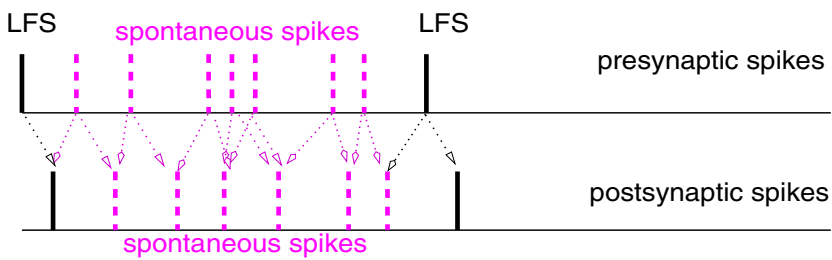

Fig. 4. Illustration of the STDP rule and the timing relationships for the LTP and LTD windows during LFS. (a) Without spontaneous activity. (b) With spontaneous pre- and very few postsynaptic spikes like in the case of the granule cells. (c) With spontaneous pre- and many postsynaptic spikes like for instance in the case of the CA1 cells.

for fewer postsynaptic spikes. This may be the explanation why it is easier to evoke homosynaptic LTD in CA1 than in the granule cells (which have very low spontaneous spiking).

The situation for CA1 neurons can change, however, when the measurements are done under anesthesia. Urethane is a widely used anesthetic for animal experiments, and the simulated experiments were done either in awake rats or in rats anesthetized with urethane. Although urethane is thought to minimally interfere with neurophysiological processes and preserves synaptic signal transmission, it has also been reported that it may increase the interspike interval, in other words decrease the rate of spontaneous firing [25]. Thus anesthesia may reduce the frequency of spontaneous spiking at both pre- and postsynaptic levels and scenario may look like in Figure 4B or even 4A and in fact either no change or even potentiation may follow the LFS. Recently, indeed it has been demonstrated that LFS evokes LTP in the CA1 area of hippocampus in urethane anesthetized rats [26].

Our future work will focus on extending the model to account for plasticity in the area CA1 and also on testing 
the robustness of the model with respect to variation of the rest of parameter values like for instance the number of input fibers/synapses belonging to each of the three pathways. Now the model cell has only 3 inputs, thus it would be interesting to experiment with hundreds of input fibers, each of which has a different jitter. This extension is prepared for with the parameter $I_{\mathrm{MPP}}=I_{\mathrm{LPP}}=I_{\mathrm{ComAs}}=150$, which represents the number of input fibers engaged in stimulation and which can be easily replaced with actual simulated fibers. We expect however, that the general scenario outlined in Figure 4 holds for any number of input fibers.

At this stage, the model generates several predictions that can be tested experimentally. First prediction is that there is a heterosynaptic LTD in the commissural/associational input accompanying the homosynaptic LTP of the whole ipsilateral perforant path. This follows directly from the simulations of three input pathways. Second prediction is that the amplitudes of LTP and LTD depend on the average postsynaptic activity of a neuron over some recent past. This is the metaplasticity assumption of the model. Third prediction is that in order to induce homosynaptic LTD one has to increase the frequency of stimulation so that the depression will actually dominate over potentiation (see Figure 4A). From this follows the fourth prediction that LFS may in some cases lead to LTP. This has indeed been observed recently in the hippocampus by Habib and Dringenberg on CA1 neurons [26].

To summarize, in this paper we have offered some new insights why it is difficult to induce homosynaptic LTD by LFS in granule cells in the dentate gyrus in vivo, while it is easy to induce the heterosynaptic LTD at the same synapses by applying HFS to the neighbouring pathway. These insights follow from the classical pair-wise STDP rule when we take into account also an ongoing spontaneous activity of neurons and metaplasticity. The main conclusion of the paper is that the ongoing input spiking activity is an important player in synaptic plasticity and that it should be included in theoretical and computational studies. We think that the stimulation protocol used for induction of plasticity plus the ongoing spontaneous activity and the phenomenon of metaplasticity are closely related and cannot be separated when studying synaptic plasticity.

\section{ACKNOWLEDGMENT}

This research was supported by the University of Otago Research Grant. I am grateful to Cliff Abraham and Peter Jedlička for many stimulating discussions.

\section{REFERENCES}

[1] W. C. Abraham, S. E. Mason-Parker, M. F. Bear, S. Webb, and W. P. Tate, "Heterosynaptic metaplasticity in the hippocampus in vivo: a BCM-like modifiable threshold for LTP," Proc. Natl. Acad. Sci. USA, vol. 98, pp. 10924-10929, 2001.

[2] V. Doyère, B. Srebro, and S. Laroche, "Heterosynaptic LTD and depotentiation in the medial perforant path of the dentate gyrus in the freely moving rat," J. Neurophysiol., vol. 77, pp. 571-578, 1997.

[3] A. J. Heynen, W. C. Abraham, and M. F. Bear, "Bidirectional modification of CA1 synapses in the adult hippocampus in vivo," Nature, vol. 381, no. 6578, pp. 163-166, 1996.
[4] B. L. McNaughton, C. A. Barnes, and P. Andersen, "Synaptic efficacy and EPSP summation in rat granule cells of rat fascia dentata studied in vitro," J. Neurophysiol., vol. 46, pp. 952-966, 1981.

[5] E. Förster, S. Zhao, and M. Frotscher, "Laminating the hippocampus," Nature Rev. Neurosci., vol.7, pp. 259-268, 2006.

[6] M. L. Errington, T. V. Bliss, G. Richter-Levin, K. Yenk, V. Doyère, and S. Laroche, "Stimulation at $1-5 \mathrm{~Hz}$ does not produce long-term depression or depotentiation in the hippocampus of the adult rat in vivo," J. Neurophysiol., vol. 74, no. 4, pp. 1793-1799, 1995.

[7] W. C. Abraham, S. E. Mason-Parker, and B. Logan, "Low-frequency stimulation does not readily cause long-term depression or depotetiation in the dentate gyrus of awake rats," Brain Res., vol. 722, pp. 217-221, 1996.

[8] S. J. Martin, "Time-dependent reversal of dentate LTP by $5 \mathrm{~Hz}$ stimulation," Neuroreport, vol. 9, no. 17, pp. 3775-3781, 1998.

[9] H. Markram, J. Lübke, M. Frotscher, and B. Sakmann, "Regulation of synaptic efficacy by coincidence of postsynaptic APs and EPSPs," Science, vol. 275, pp. 213-215, 1997.

[10] P. J. Sjöström, E.A. Rancz, A. Roth, and M. Häusser, "Dendritic excitability and synaptic plasticity," Physiol. Rev., vol. 88, pp. 769-840, 2008.

[11] Y-W. Lin, H-W. Yang, H-J. Wang, C-L. Gong, T-H Chiu, and M-Y. Min, "Spike-timing-dependent plasticity at resting and conditioned lateral perforant path synapses on granule cells in the dentate gyrus: different roles of $N$-methyl-D-aspartate and group I metabotropic glutamate receptors," Eur. J. Neurosci., vol. 23, pp. 2362-2374, 2006.

[12] L. Benuskova, and W. C. Abraham, "STDP rule endowed with the BCM sliding threshold accounts for hippocampal heterosynaptic plasticity," $J$. Comp. Neurosci., vol. 22, pp. 129-133, 2007.

[13] E. L. Bienenstock, L. N Cooper, and P. W. Munro, "Theory for the development of neuron selectivity: orientation specificity and binocular interaction in visual cortex," J. Neurosci., vol. 2, pp. 32-48, 1982.

[14] W. C. Abraham, B. Logan, A. Wolff, and L. Benuskova, "Heterosynaptic" LTD in the dentate gyrus of anesthetized rat requires homosynaptic activity," J. Neurophysiol., vol. 98, no. 2, pp. 1048-1051, 2007.

[15] E. M. Izhikevich, "Simple model of spiking neurons," IEEE Trans. Neural Networks, vol. 14, pp. 1569-1572, 2003.

[16] E. M. Izhikevich, "Which model to usefor cortical spiking neurons," IEEE Trans. Neural Networks, vol. 15, pp. 1063-1070, 2004.

[17] L. M. Frank, E. N. Brown, and M. A. Wilson, "A comparison of the firing properties of putative excitatory and inhibitory neurons from CA1 and the entorhinal cortex," J. Neurophysiol., vol. 86, pp. 2029-2049, 2005.

[18] T. Gloveli, D. Schmitz, R. M. Empson, and U. Heineman, "Frequencydependent information flow from the entorhinal cortex to the hippocampus," J. Neurophysiol., vol. 78, pp. 3444-3449, 1997.

[19] A. Kimura and C. Pavlides, "Long-term potentiation/depotentiation are accompanied by complex changes in spontaneous unit activity in the hippocampus," J. Neurophysiol., vol. 84, pp. 1894-1906, 2000.

[20] J.-P. Pfister and W. Gerstner, "Triplets of spikes in a model of spike timing-dependent plasticity," J. Neurosci., vol. 26, pp. 9673-9682, 2006.

[21] E. M. Izhikevich and N. S. Desai, "Relating STDP to BCM," Neural Comput., vol. 15, pp. 1511-1523, 2003.

[22] E. E. Clothiaux, M. Bear, and L. N Cooper, "Synaptic plasticity in visual cortex: comparison of theory with experiment," J. Neurophysiol., vol. 66, no. 5, pp. 1785-1804, 1991.

[23] L. Benuskova, R. Velayudhan, M. Armstrong-James, and F. F. Ebner, "Theory for normal and impaired experience-dependent plasticity in neocortex of adult rats," Proc. Natl. Acad. Sci. USA, vol. 98, no. 5, pp. 2797-2802, 2001.

[24] C. G. Mayr and J. Partzsch. (2010) Rate and pulse based plasticity governed by local synaptic state variables. [Online]. Available: http : //www.frontiersin.org/synaptic_neuroscience/ 10.3389/fnsyn.2010.00033/full

[25] Y. Tian, T. Lei, Z. Yang, and T. Zhang, "Urethane suppresses hippocampal CA1 neuron excitability via changes in presynaptic glutamate release and in potassium channel activity," Brain Res. Bul., vol. 87, pp. 420-426, 2012.

[26] D. Habib, and H. C. Dringenberg, "Alternating low frequency stimulation of medial septal and commissural fibers induces NMDAdependent, long-lasting potentiation of hippocampal synapses in urethaneanesthetized rats," Hippocampus, vol. 19, pp. 299-307, 2009. 